\title{
Addition of either pioglitazone or a sulfonylurea in type 2 diabetic patients inadequately controlled with metformin alone: Impact on cardiovascular events. A randomized controlled trial
}

\author{
O. Vaccaro ${ }^{a, i, *}$, M. Masulli ${ }^{a, i}$, E. Bonora ${ }^{b, i}$, S. Del Prato ${ }^{c, i}$, C.B. Giorda ${ }^{\text {, }}$,

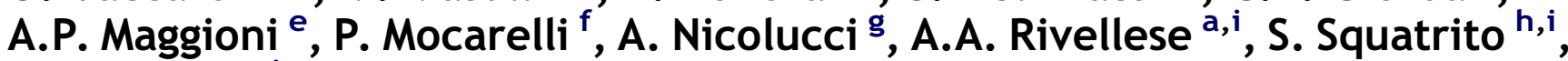 \\ G. Riccardi ${ }^{a, i}$ On behalf of the TOSCA.IT study group (Thiazolidinediones Or Sulphonylureas and Cardiovascular \\ Accidents. Intervention Trial)
}

\footnotetext{
a Department of Clinical and Experimental Medicine, "Federico II" University, Naples, Italy

${ }^{\mathrm{b}}$ Division of Endocrinology Diabetes and Metabolism, Department of Medicine, University of Verona Medical School and Azienda Ospedaliera Universitaria Integrata, Verona, Italy

' Department of Internal Medicine, University of Pisa, Pisa, Italy

dDiabetes and Metabolism Unit, ASL TORINO 5, Turin, Italy

e ANMCO Research Center, Florence, Italy

${ }^{f}$ University Milano Bicocca, Department of Laboratory Medicine, Hospital of Desio, Monza Brianza, Italy

${ }^{g}$ Department of Clinical Pharmacology and Epidemiology, Consorzio Mario Negri Sud, S Maria Imbaro, Chieti, Italy

${ }^{\mathrm{h}}$ Endocrinology Division, Department of Clinical and Molecular Biomedicine, University of Catania, Garibaldi-Nesima Hospital, Catania, Italy

'Società Italiana di Diabetologia (SID), Rome, Italy
}

Received 4 July 2012; received in revised form 27 August 2012; accepted 1 September 2012 Available online 11 October 2012

\section{KEYWORDS \\ Type 2 diabetes; \\ Cardiovascular \\ events; \\ Pioglitazone; \\ Sulfonylureas; \\ Randomized \\ Controlled Trial}

\begin{abstract}
Background and aims: Metformin is the first-line therapy in type 2 diabetes. In patients inadequately controlled with metformin, the addition of a sulfonylurea or pioglitazone are equally plausible options to improve glycemic control. However, these drugs have profound differences in their mechanism of action, side effects, and impact on cardiovascular risk factors. A formal comparison of these two therapies in terms of cardiovascular morbidity and mortality is lacking. The TOSCA.IT study was designed to explore the effects of adding pioglitazone or a sulfonylurea on cardiovascular events in type 2 diabetic patients inadequately controlled with metformin.
\end{abstract}

* Corresponding author. Department of Clinical and Experimental Medicine, "Federico II" University of Naples, Via S. Pansini 5, 80131 Naples, Italy. Tel.: +39 0817463665; fax: +390815466152.

E-mail address: ovaccaro@unina.it (0. Vaccaro). 
Methods: Multicentre, randomized, open label, parallel group trial of 48 month duration. Type 2 diabetic subjects, $50-75$ years, BMI $20-45 \mathrm{Kg} / \mathrm{m}^{2}$, on secondary failure to metformin monotherapy will be randomized to add-on a sulfonylurea or pioglitazone. The primary efficacy outcome is a composite endpoint of all-cause mortality, nonfatal myocardial infarction, nonfatal stroke, and unplanned coronary revascularization. Principal secondary outcome is a composite ischemic endpoint of sudden death, fatal and non-fatal myocardial infarction and stroke, endovascular or surgical intervention on the coronary, leg or carotid arteries, major amputations. Side effects, quality of life and economic costs will also be evaluated. Efficacy, safety, tolerability, and study conduct will be monitored by an independent Data Safety Monitoring Board. End points will be adjudicated by an independent external committee.

Conclusions: TOSCA.IT is the first on-going study investigating the head-to-head comparison of adding a sulfonylurea or pioglitazone to existing metformin treatment in terms of hard cardiovascular outcomes.

Registration: Clinicaltrials.gov ID NCT00700856.

(C) 2012 Elsevier B.V. All rights reserved.

\section{Introduction}

There is general agreement that metformin, if not contraindicated and well tolerated, should be the first agent in the treatment of type 2 diabetes [1-5], but considerable uncertainty does exists on the best therapeutic option as second line treatment after metformin failure. According to recent guidelines, in patients inadequately controlled with metformin, the addition of a sulfonylurea (SU) or pioglitazone are equally plausible options to improve glycemic control [6,7]. However, these two drugs have profound differences in their mechanisms of action, side effects, and impact on cardiovascular (CV) risk factors.

SUs have been available for a long time and are the least expensive and more widely used oral hypoglycemic agents. They stimulate insulin secretion by binding the ATP-sensitive potassium channels in the beta cells. SUs rapidly lower blood glucose, but are associated with modest weight gain, increased risk of hypoglycaemia and a secondary failure rate that exceeds other drugs [8]. In addition, the CV effects of SUs are debated. These agents bind to various degrees the ATP channels in the cardiomyocytes thus blunting the myocardial preconditioning mechanism $[9,10]$. To what extent this may impact CV morbidity and mortality is unclear. Observational studies report higher all-cause and CV mortality with SUs vs. metformin monotherapy [11-16] and in case series of coronary angioplasty, patients on SUs had higher in-hospital mortality than patients on other treatments [17]. To the contrary, data from the French registry on Acute ST and non ST-elevation Myocardial Infarction (FASTMI) have shown a better outcome in patients on SUs at admission as compared with patients on other treatments [18]. As for intervention studies, in the University Group Diabetes Program (UGDP) [19] patients receiving tolbutamide experienced more CV events than those treated with insulin, whereas in the UKPDS there is no evidence of an adverse effect of SUs on CVD outcomes [20] and more recently, in the Action in Diabetes and Vascular Disease: Preterax and Diamicron-MR Controlled Evaluation (ADVANCE) study, intensive therapy with gliclazide significantly reduced the risk of microvascular endpoint, with no detrimental effects on macrovascular events [21]. The thiazolidinediones (TZDs) are insulin sensitizing agents [22]: they lower blood glucose without causing hypoglycaemia and provide more durable glycemic effect than other drugs [8]. Pioglitazone also improves lipid profile, blood pressure, albuminuria, and inflammatory and procoagulant markers, an array of effects which may translate into better CV outcomes. Furthermore the PROACTIVE (PROspective pioglitAzone Clinical Trial In macroVascular Events) study has shown that the addition of pioglitazone to the hypoglycaemic therapy in patients with type 2 diabetes and overt macrovascular disease significantly reduced a composite end point of all-cause mortality, nonfatal MI and stroke as compared to placebo [23]. In addition the CHICAGO and PERISCOPE studies have shown that pioglitazone, as compared with glimepiride, reduces the progression of carotid intima-media thickness [24] and the progression of intracoronary atherosclerosis [25]. Recognized side effects of pioglitazone, which may restrain a more common use, include unwanted weight gain, fluid retention leading to edema and/or heart failure in predisposed individuals, increased risk of bone fractures and possibly bladder cancer [26,27].

Due to the paucity of experimental evidence, the algorithms for managing glycemia recommended by the several professional associations and scientific societies are largely based on the opinion of experts and are not fully concordant [1-7]. In particular, the CVD effects of glucoselowering agents remain debated. The Thiazolidinediones Or Sulphonylureas and Cardiovascular Accidents. Intervention Trial (TOSCA.IT) study was designed and initiated to compare the effects of the addition of SUs vs. pioglitazone on $\mathrm{CV}$ events in patients with type 2 diabetes inadequately controlled with metformin monotherapy. Durability of glucose control, side effects, quality of life and costs will also be evaluated.

\section{Methods}

\section{Research design}

The study (protocol number FARM6T9CET), registered in the clinicaltrials.gov with ID NCT00700856, is a multicentre, 
parallel-group trial of 48 months duration. A Prospective Randomized Open Blinded End-Point Evaluation (PROBE) design is employed. The protocol has been approved by the Ethics Review Committee/Insitutional Review Board of the Coordinating Centre and of each participating centre. The study is carried out in accordance with the Declaration of Helsinki and the Good Clinical Practice guidelines. Written informed consent must be obtained from participants before beginning any protocol-specific procedure and the participants are informed of their right to withdraw from the study at any time. Standard procedures for assuring full respect of privacy are undertaken. The conduct of each participating center is monitored by regular visits of professional monitors.

\section{Efficacy end points}

The primary efficacy outcome is a composite of all-cause mortality, nonfatal myocardial infarction (MI) (including silent MI), nonfatal stroke, unplanned coronary revascularization. The principal secondary outcome is a composite ischemic end point of sudden death, fatal and non-fatal MI (including silent $\mathrm{MI}$ ), fatal and nonfatal stroke, major leg amputation (above the ankle), endovascular or surgical interventions on the coronary, leg or carotid arteries.

Other secondary outcomes are:

1) A composite $C V$ endpoint including the primary endpoint plus hospitalization for heart failure, endovascular or surgical intervention on the coronary, leg or carotid arteries, incident angina or intermittent claudication;

2) All cases of heart failure;

3) A microvascular composite endpoint including: incident macroalbuminuria, or doubling of baseline plasma creatinine, or a creatinine clearance reduction of $20 \mathrm{ml} / \mathrm{min} / 1.73 \mathrm{~m}^{2}$ or plasma creatinine $>3.3 \mathrm{mg} / \mathrm{dl}$, or dialysis;
4) Glucose control (changes from baseline in HbA1c, time to failure of oral hypoglycaemic therapy, defined as $\mathrm{HbA} 1 \mathrm{C}>\mathbf{8 . 0 \%}$ on two occasions three months apart);

5) Changes from baseline of the CV risk factors profile (lipids, blood pressure, microalbuminuria, inflammation markers, waist circumference).

A Clinical Endpoints Committee (CEC) of expert clinicians (cardiologists, diabetologists, internal medicine specialists and epidemiologists), blind to study medication assignment, is reviewing and adjudicating the outcomes included in the primary endpoint plus all the cases of heart failure and revascularization according to predefined criteria.

\section{Visits and procedures}

The eligibility criteria are listed in Table 1 . Eligible patients are randomized to one of two treatments (Fig. 1): metformin + sulfonylurea (glibenclamide $5 \mathrm{mg}$ or gliclazide $30 \mathrm{mg}$ or glimepiride $2 \mathrm{mg}$ used according to local practice) or metformin + pioglitazone $(15 \mathrm{mg})$. Treatment is centrally assigned by telephone after verification of eligibility. The treatment allocation schedule is computer generated in blocks and stratified according to centre and previous CV events. The investigators are masked to the randomization sequences.

An outline of the study procedures is given in Table 2. At screening the study aims and procedures are extensively discussed with the patients, a written informed consent is obtained and the inclusion/exclusion criteria are assessed. Patients deemed eligible and willing to participate are enrolled. At baseline (randomization visit) the inclusion/ exclusion criteria are reassessed, a complete medical history, including prior CV events and use of medications, is recorded; a complete physical examination, including standardised measurements of blood pressure, body weight, height, waist and hip circumference and a standard

Table 1 Overview of inclusion/exclusion criteria.

\begin{tabular}{ll}
\hline Inclusion criteria & Exclusion criteria \\
\hline - Type 2 diabetes of at least 2 years duration & - Type 1 diabetes \\
- Males and females, age $50-75$ years & - Previous treatment with TDZs within the last six months \\
- BMI $20-45 \mathrm{Kg} / \mathrm{m}^{2}$ & - Contraindication/intolerance to metformin or SUs or TZDs \\
- Stable treatment for the last two & - Documented coronary or cerebrovascular events within \\
months with metformin in & the previous 3 months \\
monotherapy at $2 \mathrm{~g} / \mathrm{day}$ & - Serum creatinine $>1.5 \mathrm{mg} / \mathrm{dl}$ \\
- HbA1c $\geq 7.0 \%$ and $\leq 9.0 \%$ & - History of congestive heart failure, NYHA class I or higher \\
& - Chronic use of glucocorticoids \\
& - Ischemic ulcer or gangrene of lower extremities \\
& - Liver cirrosis or severe hepatic dysfunction (ALT $>2.5$ times \\
& the upper normal limit) \\
& - Pregnancy or breast feeding \\
& - Cancer, substance abuse, or any health problem that may \\
& interfere with the compliance to the study protocol or limit \\
& life expectancy \\
\hline
\end{tabular}

BMI: body mass index; HbA1c: glycated hemoglobin; SUs: sulfonylureas; TZDs: thiazolidinediones; NYHA: New York Heart Association; ALT: Alanine aminotransferase. 
Table 2 Overview of the study assessments.

\begin{tabular}{|c|c|c|c|c|c|c|c|c|}
\hline \multirow[t]{2}{*}{ Visit } & \multirow[t]{2}{*}{ Screening } & \multirow[t]{2}{*}{ Baseline } & \multicolumn{4}{|c|}{ Year 1} & \multicolumn{2}{|l|}{ Years 2-4 } \\
\hline & & & $1 \mathrm{mo}$ & $3 \mathrm{mo}$ & $6 \mathrm{mo}$ & $12 \mathrm{mo}$ & Semiannually & Annually \\
\hline $\begin{array}{l}\text { Inclusion/exclusion } \\
\text { criteria assessment }\end{array}$ & $x$ & $x$ & & & & & & \\
\hline Informed consent obtainment & $x$ & & & & & & & \\
\hline Clinical history & $x$ & $x$ & $x$ & $x$ & $x$ & $x$ & $x$ & $x$ \\
\hline Anthropometry & $x$ & $x$ & $x$ & $x$ & $x$ & $x$ & $x$ & $x$ \\
\hline Clinical examination & $x$ & $x$ & $x$ & $x$ & $x$ & $x$ & $x$ & $x$ \\
\hline ECG & & $x$ & & & & $x$ & & $x$ \\
\hline Food frequency questionnaire & & $x$ & & & & $x$ & & $x$ \\
\hline Quality of life questionnaire & & $x$ & & & $x$ & & & $x$ \\
\hline Economic questionnaire & & $x$ & & & $x$ & & & $x$ \\
\hline \multicolumn{9}{|l|}{ Biochemistry } \\
\hline $\mathrm{HbA1c}$ & & $x$ & & & $x$ & $x$ & $x$ & $x$ \\
\hline Lipids & & $x$ & & & & $x$ & & $x$ \\
\hline Creatinine & & $x$ & & & & $x$ & & $x$ \\
\hline Microalbuminuria & & $x$ & & & & $x$ & & $x$ \\
\hline PCR & & $x$ & & & & $x$ & & $x$ \\
\hline $\begin{array}{l}\text { Urinalysis and assessment } \\
\text { of hematuria }^{a}\end{array}$ & & $x$ & & & $x$ & $x$ & $x$ & $x$ \\
\hline Hypoglycaemia & & $x$ & $x$ & $x$ & $x$ & $x$ & $x$ & $x$ \\
\hline Compliance & & $x$ & $x$ & $x$ & $x$ & $x$ & $x$ & $x$ \\
\hline Endpoint events & & $x$ & $x$ & $x$ & $x$ & $x$ & $x$ & $x$ \\
\hline Adverse events & & $x$ & $x$ & $x$ & $x$ & $x$ & $x$ & $x$ \\
\hline
\end{tabular}

Plasma lipids include: total cholesterol, HDL cholesterol and triglycerides.

a Starting from January 2012.

12-lead ECG, is performed. Fasting blood samples and a morning spot urine sample are collected for biochemical analyses (see Table 2). Dietary habits are assessed using a validated food frequency questionnaire - the Italian version of the European Prospective Investigation into Cancer and Nutrition (EPIC) questionnaire. Quality of life

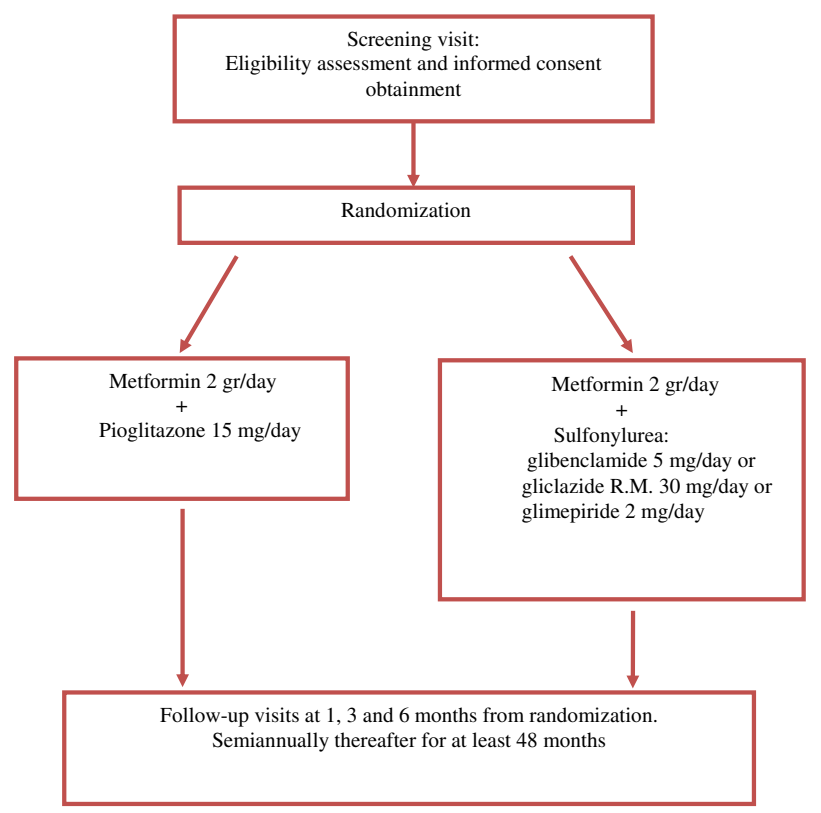

Figure 1 Flow chart of the study design. and economic costs will also be assessed with the use of standard questionnaires. All participants are instructed to perform home glucose monitoring (one full day glucose profile per week) and to record the number and severity of hypoglycaemic events.

Follow-up is scheduled at 1,3 , and 6 months after randomization and biannually thereafter (Table 2). At each visit adherence to the study protocol is assessed, information on the occurrence of any adverse or endpoint event(s) is collected. Anthropometry, blood pressure and heart rate are measured. Since January 2012, due to regulatory concerns regarding pioglitazone and the risk of bladder cancer, macroscopic hematuria is also assessed at each visit. $\mathrm{HbA} 1 \mathrm{C}$ is measured semiannually. A standard 12-lead ECG, fasting plasma lipids (total cholesterol, HDL cholesterol, triglycerides), creatinine, high sensitivity C-reactive protein (hsCRP), and urinary excretion of albumin and creatinine are performed annually. The patient's home glucose readings and the records of hypoglycaemia are reviewed at each visit. The metformin dose remains constant ( $2 \mathrm{~g} /$ day) throughout the study. The add-on drugs will be up-titrated at any follow-up visit, if necessary, based on home glucose monitoring (i.e fasting glucose $>120 \mathrm{mg} / \mathrm{dl}$ or post prandial glucose $>160 \mathrm{mg} / \mathrm{dl}$ in more than $50 \%$ of the home glucose readings performed over the last 8 weeks period). The maximum daily dose is $15 \mathrm{mg}$ for glibenclamide, $120 \mathrm{mg}$ for gliclazide, $6 \mathrm{mg}$ for glimepiride, and $45 \mathrm{mg}$ for pioglitazone. If, despite the maximal daily dose of the drugs has been reached, blood glucose control is still unsatisfactory, adherence to treatments is assessed, lifestyle recommendations are reinforced and $\mathrm{HbA} 1 \mathrm{c}$ is re- 
evaluated after three months. A confirmed $\mathrm{HbA1C}>8.0 \%$, will lead to add on a bed-time injection of basal insulin (glargine) and prandial rapid acting insulin boluses, if glucose control is still unsatisfactory. Insulin titration is performed according to a pre-defined algorithm based on self-monitored fasting capillary glucose [28].

Concomitant medications are allowed throughout the study. Initiation and dose adjustments of antihypertensive, lipid-lowering and antiplatelet agents are made according to current guidelines [7] and clinical judgment. The trade name and dosage of all medications are recorded at each visit. Ad hoc developed electronic Case Report Forms (CRFs) are used for data collection, data is web-transmitted to the Data Monitoring Centre where the forms are checked for missing or incoherent data. An electronic data base is being created using a standardized procedure for data input.

\section{Laboratory measurements}

A fasting blood sample is obtained in the morning, immediately centrifuged and serum is aliquoted; a spot urine sample is also collected and aliquoted. The samples are transferred within two days, under appropriate conditions, to the central laboratory and processed on arrival. Extra samples are stored $\left(-80^{\circ} \mathrm{C}\right)$ for future analyses. Glycated hemoglobin is measured by HPLC (ion-exchange chromatography) using an automatic analyzer (Adams $A_{1 c}$ HA-8160, Menarini). Lipids, plasma and urine creatinine, and albuminuria are measured on the automatic analyzer MODULAR SWA (Roche Diagnostics, Italy) respectively by enzymaticcolorimetric methods, ECLIA and immunoturbidimetry. CRP is determined by a high sensitive immunonephelometric method (CardioPhase hs CRP, SIEMENS) on the BN ${ }^{\top M}$ II System (SIEMENS). Brain Natriuretic peptide (NT-proBNP) can be measured centrally at any time, according to the researchers judgment, in case signs or symptoms suggestive of heart failure develop. Any other test judged useful from the clinical stand point is performed at each centre.

\section{Safety assessment}

The study drugs are largely used for the treatment of diabetes, no additional risk, besides the known side effects of the drugs, is envisaged. The occurrence of adverse events (hypoglycaemic events, weight gain, peripheral edema, heart failure episodes, etc.) is strictly monitored. Hypoglycaemia is defined as a documented glucose value lower than $60 \mathrm{mg} / \mathrm{dl}$ and graded as moderate (not requiring help for treatment) or severe (requiring assistance for treatment or associated with loss of consciousness or requiring glucagon or endovenous glucose for treatment).

Patients will stop the study medications if any of the followings occurs: alanine amino transferase increases three times from baseline on two consecutive occasions, one month apart; heart failure, evaluated according to the American Heart Association and the American Diabetes Association consensus on glitazones and heart failure [29], or any other medical condition(s) that contraindicates the use of the study medication(s). In addition, in the pioglitazone arm, treatment will be withdrawn if macroscopic hematuria of unknown origin occurs at any time or bladder cancer is diagnosed. Patients who are withdrawn from the study medications enter a follow-up observational period following the scheduled protocol visits. An external Data Safety and Monitoring Board (DSMB) will monitor safety throughout the study and review the critical efficacy endpoints.

\section{Pharmacoeconomic evaluation}

The health status and pharmacoeconomic outcomes are assessed by a standard questionnaire (EQ 5D 5 levels). Direct and indirect costs of the two treatment regimens will be evaluated; the cost-effectiveness analysis (CEA), costconsequence analysis (CCA) and cost-minimization analysis (CMA) will be conducted as appropriate.

\section{Sample size estimation}

The sample size is estimated to detect a reduction in the risk of events of $20 \%(H R=0.80$; metformin + pioglitazone vs. metformin + SU) with a statistical power of $80 \%$ and a $p<0.05$, one tail. The estimated occurrence rate of the primary end point is $3.5 \%$ per annum [30-32] and the estimated loss to follow-up is $5 \%$. The efficacy analysis will be event driven. Given these assumptions, a total of 3371 patients will be enrolled and a total of 498 events have to be reached to complete the study. The planned follow-up is 4 years; however results of recent trials have shown that the rate of occurrence of the primary end point may actually be lower than we anticipate, therefore a longer follow-up may be necessary to reach the needed number of events.

\section{Statistical analysis}

The trial is event driven and the efficacy analysis will be conducted according to intention to treat. Based on the number of occurring events, individual components of the primary and secondary composite endpoint will also be analysed.

Incidence rates will be evaluated using Kaplan-Meier survival curves that will be compared (metformin + pioglitazone vs. metformin $+\mathrm{SU}$ ) using logrank analysis. Treatment efficacy will be assessed by multivariate analyses using Cox's regression.

Other secondary analyses will include the evaluation of efficacy of metformin + pioglitazone vs. metformin + SU on pre-defined secondary end-points. Side effects, direct and indirect costs will also be compared in the two study arms. Incidence and severity of hypoglycaemia will be compared between arms using a Poisson regression model. Subgroups analyses will be performed according to gender, BMI and prior cardiovascular events.

\section{Organizational characteristics}

The study is conducted at Diabetes Clinics by diabetes specialists (a complete list of the study participants is reported in Appendix). More than one hundred clinics distributed all over Italy are currently participating in the 
study. Screening and follow-up visits are performed according to a standard protocol described in detail in the manual of operation (MOP). Prior to the initiation of the study, the investigators attended training and standardization sessions in order to minimize inter-observer variability.

The Coordinating Centre is based at the Department of Clinical and Experimental Medicine of the "Federico II" University of Naples, Italy, and is responsible for the preparation of the study protocol, the Manual of Operations (MOP) and the organization of the training sessions for the field investigators.

The Centre for the Data Monitoring and Randomization, based at the Consorzio Mario Negri Sud in Santa Maria Imbaro $(\mathrm{CH})$, provides a centralized telephone randomization system for the patients allocation to study medications, and is responsible for data management and analyses.

The ECG reading will be performed at the Centro Studi ANMCO in Florence by certified readers blinded to treatment arm and according to a standard protocol.

The biochemical analyses are performed at the Department of Laboratory Medicine, Desio Hospital, MonzaBrianza, Italy.

The food frequency questionnaire is produced and electronically read at REGGIANI S.p.A., Varese, Italy; nutrients, food groups, glycemic index, glycemic load and oxidative capacity will be calculated at the Nutritional Epidemiology Unit of the Department of Preventive \& Predictive Medicine, Fondazione IRCCS Istituto Nazionale dei Tumori, Milan, with the use of an ad hoc developed software.

The Pharmacoeconomic and Pharmacovigilance Units are based at the "Federico II" University of Naples, Italy.

\section{Preliminary data}

To date 2450 patients have been enrolled (i.e. $73 \%$ of the total sample required). End of recruitment is planned within June 2013. The general characteristics of the participants are given in Table 3. Overall they are middle aged, obese, with a mean diabetes duration of $8.6 \pm 5.8$ years, a mean $\mathrm{HbA} 1 \mathrm{C}$ of $7.7 \pm 0.5 \%$. On average, lipids and blood pressure are well controlled; $56 \%$ are on lipid lowering drugs, $68 \%$ report taking antihypertensive medications, $42 \%$ use antiplatelet medications and $10.4 \%$ report a prior $\mathrm{CV}$ event.

\section{Discussion}

In clinical practice, the beneficial effects of the various hypoglycaemic treatments must be considered not only in relation to their glucose lowering effects (reduction of HbA1c, time to failure of glucose control), but also in relation to their impact on the risk for the long term complications of diabetes. In addition the benefits have to be balanced against safety (drug-specific side effects, risk of hypoglycaemias, effects on body weight, etc.).

So far, no study has provided a direct (head to head) comparison of different anti-diabetic drugs on long term complications. As a consequence, the several compounds (SUs, pioglitazone, glinides, DPP-4 inhibitors, etc.) have been indicated by current guidelines and expert recommendations as equally plausible options when metformin
Table 3 General characteristics of the study participants $(n=2450)$.

\begin{tabular}{ll}
\hline & $M \pm \mathrm{SD}$ or $n(\%)$ \\
\hline Age & $62.8 \pm 6.6$ \\
Males $n(\%)$ & $1318(57.4)$ \\
BMI $\left(\mathrm{kg} / \mathrm{m}^{2}\right)$ & $30.3 \pm 4.4$ \\
Waist circumference (cm) & $104.3 \pm 11.1$ \\
Diabetes duration (years) & $8.6 \pm 5.8$ \\
HbA1c\% & $7.7 \pm 0.5$ \\
Total cholesterol (mmol/l) & $4.68 \pm 0.95$ \\
HDL cholesterol (mmol/l) & $1.20 \pm 0.37$ \\
LDL cholesterol (mmol/l) & $2.69 \pm 0.82$ \\
Triglycerides (mmol/l) & $1.74 \pm 0.94$ \\
Systolic blood pressure (mmHg) & $134.2 \pm 14.9$ \\
Diastolic blood pressure (mmHg) & $79.7 \pm 8.5$ \\
Microalbuminuria & $467(20)$ \\
Antihypertensive treatment & $1567(68)$ \\
Antiplatelet treatment & $953(42)$ \\
Hypolipidemic treatment & $1280(56)$ \\
Prior CV event(s) & $238(10.4)$ \\
\hline
\end{tabular}

monotherapy fails, despite the fact that mechanisms of action are different, durability is not superimposable, safety profile and costs are not identical $[6,7]$.

Comparative effectiveness research, particularly with regard to CV outcomes, has a high priority in order to allow evidence based medical decisions, improve public health and optimize cost effectiveness in the management of type 2 diabetes.

The Thiazolidinediones Or Sulphonylureas and Cardiovascular Accidents. Intervention Trial (TOSCA.IT study) is the first trial designed as a head-to-head comparison of two hypoglycaemic strategies on CV endpoints and is also the only ongoing study that evaluates the potential benefits of TZDs on cardiovascular disease, as the TIDE (Thiazolidinedione Intervention With Vitamin D Evaluation) has been terminated prematurely owing to concerns on the $\mathrm{CV}$ safety of rosiglitazone [33].

The TOSCA.IT study will also shed light on other relevant issues, such as glucose-lowering effects, time to failure of oral hypoglycaemic therapy, quality of life, side effects and economic costs, including the costs of adverse effects, particularly hypoglycaemia. This information is lacking and is strongly needed to allow an evidence based choice for the management of type 2 diabetes.

\section{Funding}

The study is supported by the Italian Medicines Agency (AIFA) within the Independent Drug Research Program contract no. FARM6T9CET and by Diabete Ricerca, the no profit Research Foundation of the Italian Diabetes Society (SID).

\section{Duality of interest}

O Vaccaro, M Masulli, A Nicolucci, CB Giorda, P Mocarelli, S Squatrito, AA Rivellese and $G$ Riccardi declare that they have no duality of interest associated with this manuscript. 
E Bonora is a member of the Italian Takeda Advisory Board and has received a research grant from Takeda in the last 2 years. S Del Prato has served on advisory panels for Novartis Pharmaceuticals, Merck \& Co., Roche Pharmaceuticals, Eli Lilly and Co., Boehringer Ingelheim, Novo Nordisk, Takeda Pharmaceuticals, Intarcia Therapeutics Inc., and Astra Zeneca and, as speaker from Novartis, Sanofi-aventis, Eli Lilly and Co. and Bristol-Myers Squibb. Research support was funded by Merck\&Co., Novo Nordisk, Takeda Pharmaceuticals, Novartis Pharmaceuticals. AP Maggioni is a member of the Steering Committee of trials sponsored by Sanofi-Aventis and Eli Lilly.

\section{Contribution statement}

Vaccaro, M Masulli, E Bonora, S Del Prato, AA Rivellese, S Squatrito, G Riccardi were involved in the conception and design of the study protocol, drafting of the article and final approval of the version to be published. A Nicolucci was involved in the design of the study and was responsible for data analysis. CB Giorda, AP Maggioni, P Mocarelli revised the manuscript critically providing important intellectual content and final approval of the version to be published.

\section{Appendix A}

Executive Committee: Gabriele Riccardi, Federico II University of Naples, Italy; Enzo Bonora, University of Verona, Italy; Stefano Del Prato, University of Pisa, Italy; Carlo B. Giorda, ASL Torino 5, Turin Italy; Aldo Pietro Maggioni, ANMCO Research Center, Florence, Italy; Paolo Mocarelli, Desio Hospital, Monza-Brianza, Italy; Antonio Nicolucci, Consorzio Mario Negri Sud, S. Maria Imbaro $(\mathrm{CH})$, Italy; Angela A. Rivellese, Federico II University of Naples, Italy; Sebastiano Squatrito, Garibaldi-Nesima Hospital, Catania, Italy; Olga Vaccaro, Federico II University of Naples, Italy. Coordinating Center: Olga Vaccaro, Maria Masulli, Nadia Garofalo, Federico II University of Naples, Italy. Data Safety Monitoring Board. Committee Members: Eleuterio Ferrannini (Chair), Università di Pisa, Italy; Barbara Howard, Medstar Research Institute, Washington, USA; Eva Gerdts, University of Bergen, Norway; Giuseppina Imperatore, CDC, Atlanta, USA; Luigi Tavazzi, GVM Care\&Research, Cotignola (RA), Italy; Fabio Pellegrini,Consorzio Mario Negri Sud, S. Maria Imbaro, $(\mathrm{CH})$ Italy. Clinical Event Committee. Gianna Fabbri (Coordinator), Centro Studi ANMCO, Florence, Italy; Giacomo Levantesi (Cardiologist), Ospedale San Pio da Pietralcina, Vasto; Fabio Turazza (Cardiologist), Ospedale Niguarda Ca' Grande di Milano; Sandro Gentile (Diabetologist), Second University of Naples, Italy; Salvatore Panico (Internist), Federico II University of Naples, Italy; Massimo Porta (Internist), Ospedale Le Molinette, University of Torino, Italy.

Central Laboratory. Paolo Mocarelli, Paolo Brambilla, Stefano Signorini, Fabrizio Cappellini, Chiara Parma, Department of Laboratory Medicine, Desio Hospital, MonzaBrianza, Italy. Data managing. Antonio Nicolucci, Daniela D’Alonzo, Barbara Di Nardo, Sonia Ferrari, Monica Franciosi, Romina Pecce, Miriam Valentini. Consorzio Mario Negri Sud, S. Maria Imbaro, (CH) Italy.
On site monitoring. Martina Ceseri, Francesca Bianchini, Ester Baldini, Antonio Atzori. ANMCO Research Center, Florence, Italy.

\section{Participating centers}

Ancona - INRCA - IRCCS Institute- Principal Investigator: Massimo Boemi. Co-investigators: Federica D’Angelo, Roberto Giansanti

Arezzo - Ospedale San Donato - Principal Investigator: Lucia Ricci. Co-investigator: Anna Ranchelli

Atri (TE) - Ospedale di Atri - Principal Investigator: Paolo Di Berardino. Co-investigator: Emanuela Cannarsa

Bari - Policlinico Consorziale - Principal Investigator: Francesco Giorgino. Co-investigator: Mariangela Manicone, Lucia Tarantino

Bergamo - Ospedali Riuniti di Bergamo - Principal Investigator: Roberto Trevisan. Co-investigator: Cristiana Scaranna

Bologna - Policlinico S. Orsola Malpigli - Principal Investigator: Gabriele Forlani. Co-investigator: Luca Montesi

Campobasso - Presidio Ospedaliero A.S.RE.Molise - Principal Investigator: Antimo Aiello. Co-investigator: Angelina Barrea

Catania - Garibaldi-Nesima Hospital- Principal Investigator: Sebastiano Squatrito. Co-investigators: Tiziana Sinagra, Sara Longhitano

Catanzaro - "Magna Graecia" University - Principal Investigators: Giorgio Sesti, Agostino Gnasso. Co-investigators: Claudio Carallo, Caterina Scicchitano

Cesena - M. Bufalini Hospital - Principal Investigator: Costanza Santini. Co-investigators: Giovanni Calbucci, Raffaella Ripani

Chiavari (GE) - ASL Chiavarese - Principal Investigator: Laura Corsi. Co-investigator: Simona Corsi

Chieri (TO) - Ospedale Maggiore - Principal Investigator: Carlo Bruno Giorda. Co-investigator: Francesco Romeo

Cosenza - Azienda Sanitaria Provinciale - Principal Investigator: Vincenzo Asprino. Co-investigator: Giovanna Donnarumma.

Eboli (SA) - District 64 - Principal Investigator: Biagio Tizio, Co-investigator: Gennaro Clemente

Ferrara - Arcispedale "S.Anna"- Principal Investigator: Franco Tomasi. Co-investigator: Nicoletta Dozio

Firenze - Careggi - Pontenuovo Hospital - Principal Investigator: Edoardo Mannucci. Co-investigator: Caterina Lamanna

Foggia - OORR of Foggia - Principal Investigator: Mauro Cignarelli. Co-investigators: Olga La Macchia, Stefania Fariello

Genova - DISEM - Università di Genova - Principal Investigator: Renzo Cordera. Co-investigator: Chiara Mazzucchelli

Lanciano (CH) - Ospedale di Lanciano - Principal Investigators: Mario Pupillo, Ercole D'Ugo. Co-investigator: Antonella Zugaro, Angela Bosco, Angelo De Luca

L'Aquila - Ospedale San Salvatore - Principal Investigator: Rossella Iannarelli. Co-investigators: AnnaElisa Giuliani, Lorella Polidoro, Alessandra Sperandio, Filomena Sciarretta

Latina - Ospedale S.Maria Goretti di Latina - Principal Investigator: Buzzetti Raffaella. Co-investigators: Chiara Venditti

Livorno - ASL 6 Livorno - Principal Investigator: Graziano Di Cianni. Co-investigator: Chiara Goretti 
Massa Carrara - USL 1 - Principal Investigator: Maria A. Dolci. Co-investigator: Laura Bruselli, Mary Mori, Fabio Baccetti, Giovanna Gregori

Matera - Ospedale di Matera- Principal Investigator: Angelo Venezia

Messina - Policlinico G. Martino - Principal Investigator: Domenico Cucinotta. Co-investigator: Basilio Pintaudi

Messina- Ospedali Riuniti Papardo- Piemonte" di Mes sina- Principal Investigator: Francesco Ragonese. Co-investigator: Pietro Pata

Milano - San Raffaele Diabetes Research Institute Principal Investigator: PierMarco Piatti. Co-investigator: Pietro Luccotti

Milano - Ospedale Maggiore Ca' Grande in cooperation with Ospedale di Treviglio - Principal Investigator: Emanuela Orsi

Milano - Ospedale Niguarda Ca' Granda”- Principal Investigator: Matteo Bonomo. Co-investigator: Laura Menicatti

Napoli - Università "Federico II" - Internal Medicine Unit - Principal Investigator: Angela Albarosa Rivellese. Coinvestigators: Anna Amelia Turco, Ornella Ciano, Pasquale Vallefuoco, Gerardo Corigliano.

Napoli - ASL NA2 - in cooperation with Università di Napoli "Federico II" - Co-investigators: Carmine Pentangelo, Ettore Petraroli, Pasquale Auletta, Ornella Carbonara, Giuseppe Capobianco

Napoli - ASL NA3 - in cooperation with Università di Napoli "Federico II" - Co-investigators: Gennaro Caiazzo, Bruno Angiulli, Giuseppina De Simone, Cutolo Michele, Valeria Mastrilli, Giuseppe Nunziata, Geremia Romano, lole Gaeta, Tommasina Sorrentino

Napoli - Università "Federico II" - Diabetes Unit Principal Investigator: Ciro lovine. Co-investigators: Giovanna Donnarumma, Francesca Nappi

Napoli - Seconda Università - Principal Investigator: Giuseppe Paolisso. Co-investigators: Maria Rosaria Rizzo

Padova - Policlinico di Padova- Principal Investigator: Angelo Avogaro. Co-investigator: Monica Vedovato

Padova - Complesso Socio-Sanitario ai Colli- Principal Investigator: Annunziata Lapolla. Co-investigators: Giovanni Sartore, Silvia Burlina, Nino Cristiano Chilelli

Palermo - Università degli Studi di Palermo - Principal Investigators: Aldo Galluzzo†, Carla Giordano. Co-investigator: Vittoria Torregrossa

Parma - AOU di Parma - Principal Investigator: Leone Arsenio. Co-investigators: Elisabetta Dall'Aglio, Federico Cioni, Massimiliano Babini, Giovanni Moncastroppa

Perugia - Università di Perugia - Principal Investigator: Gabriele Perriello. Co-investigator: Alessia Timi

Pescara - Ospedale Civile di Pescara - Principal Investigator: Agostino Consoli. Co-investigator: Federica Ginestra

Piacenza - Ospedale Guglielmo da Saliceto - Principal Investigator: Donatella Zavaroni. Co-investigator: Fabio Calzoni

Pisa - Ospedale di Cisanello - Principal Investigator: Stefano Del Prato. Co-investigators: Roberto Miccoli, Cristina Bianchi, Savvina Politi

Pistoia - Spedali Riuniti di Pistoia - Principal Investigator: Roberto Anichini. Co-investigator: Anna Tedeschi

Potenza - Rete ASP - Potenza - Maratea - Matera Villa d'Agri - Principal Investigator: Giuseppe Citro. Coinvestigators: Armando Zampino, Sinisi Rosa, Maria
Natale, Concetta Lombardi Giocoli, Angelo Venezia, Enzo Caruso

Praia a Mare (CS) - Presidio ospedaliero di Praia a Mare Principal Investigator: Luciano Tramontano. Co-investigator: Alessia Imbroinise, Carmelo Di Perna

Prato - Ospedale di Prato - Principal Investigator: Maria Calabrese. Co-investigator: Alessia Zogheri

Ravenna - AUSL Provincia di Ravenna - Principal Investigator: Paolo Di Bartolo. Co-investigator: Alessandra Luberto

Rimini - Ospedale Infermi di Rimini - Principal Investigator: Giorgio Ballardini. Co-investigators: Anna Carla Babini, Chiara Zannoni

Roma - Azienda Ospedaliera S. Andrea - Principal Investigator: Giuseppe Pugliese. Co-investigators: Laura Salvi, Giulia Mazzitelli, Alessandra Zappaterreno

Roma - Ospedale Fatebenefratelli in cooperation with Azienda Ospedaliera S. Andrea di Roma- Principal Investigator: Simona Frontoni. Co-investigators: Alessia Ventricini

Roma - Policlinico di Tor Vergata - Principal Investigator: Davide Lauro. Co-investigators: Angelica Galli, Maria Elena Rinaldi

Roma - Ospedale Pertini - Principal Investigator: Sergio Leotta. Co-investigators: Lucia Fontana

Roma - Campus Biomedico in cooperation with Ospedale S. Maria Goretti di Latina- Principal Investigator: Paolo Pozzilli

Roma - Policlinico Umberto I in cooperation with Azienda Ospedaliera S. Andrea di Roma- Principal Investigator: Frida Leonetti

Roma - Policlinico Umberto I in cooperation with Ospedale Pertini di Roma - Principal Investigator: Susanna Morano, Sebastiano Filetti

San Giovanni Rotondo - Ospedale Casa Sollievo della Sofferenza - Principal Investigator: Salvatore De Cosmo. Coinvestigators: Simonetta Bacci, Antonio Pio Palena

Salerno - ASL di Salerno in cooperation with Università di Napoli "Federico II" - Co-investigators: Pasqualino Calatola, Gelsomina Capuano, Umberto Amelia

Siena - Policlinico "Le Scotte" - Principal Investigator: Francesco Dotta. Co-investigators: Elisa Guarino, Elena Ceccarelli

Spoleto- Ospedale San Matteo degli infermi- Principal Investigator: Carlo Lalli. Co-investigators: Maura Scarponi

Terracina (LT) - Polo Sud ASL di Latina - Principal Investigator: Elisa Forte. Co-investigators: Stella Potenziani

Torino - Az. Ospedaliera S.Giovanni Battista di Torino Principal Investigator: Paolo Cavallo Perin. Co-investigators: Saverio Marena, Chiara Zucco, Massimo Perotto

Treviglio - Ospedale di Treviglio - Principal Investigator: Antonio Bossi. Co-investigators: Marcello Filopanti

Udine - Azienda Ospedaliero Universitaria "S.Maria della Misericordia" - Endocrinology Unit - Principal Investigator: Franco Grimaldi. Co-investigators: Laura Tonutti

Udine - Azienda Ospedaliero Universitaria "S.Maria della Misericordia" - Diabetes Unit - Principal Investigator: Alessandro Cavarape

Verona - Ospedale Civile di Verona - Principal Investigator: Enzo Bonora. Co-investigators: Massimo Cigolini, Isabella Pichiri, Corinna Brangani, Elena Tomasetto

Verona - Ospedale Civile "G. Fracastoro" di San Bonifacio - Principal Investigator: Claudio Capra Co-investigators: Massimo Cigolini 


\section{References}

[1] IDF clinical guidelines task force global guideline for type 2 diabetes. Brussels: International Diabetes Federation; 2005.

[2] Rodbard HW, Jellinger PS, Davidson JA, Einhorn D, Garber AJ, Grunberger $G$, et al. Statement by an American association of clinical endocrinologists/American college of endocrinology consensus panel on type 2 diabetes mellitus: an algorithm for glycemic control. Endocr Pract 2009;15:540-59.

[3] Berard LD, Booth G, Capes S, Quinn K, Woo V. Canadian diabetes association 2008 clinical practice guidelines for the prevention and management of diabetes in Canada. Can $\mathrm{J}$ Diabetes 2008;32:S1-201.

[4] NICE. Type 2 diabetes: the management of type 2 diabetes: NICE clinical guideline 87: national institute for health and clinical excellence; 2009.

[5] National evidence based guideline for blood glucose control in type 2 diabetes. Prepared by The Boden Institute of Obesity, Nutrition and Exercise, The University of Sydney In collaboration with The Diabetes Unit, Menzies Centre for Health Policy, The University of Sydney. For the Diabetes Australia Guideline Development Consortium. Available from: http://www.nhmrc.gov.au/_files_nhmrc/file/publications/ synopses/di19-diabetes-blood-glucose-control.pdf.

[6] Inzucchi SE, Bergenstal RM, Buse JB, Diamant M, Ferrannini E, Nauck $M$, et al. Management of hyperglycaemia in type 2 diabetes: a patient-centered approach. Position statement of the American diabetes association (ADA) and the European association for the study of diabetes (EASD). Diabetologia 2012;55(6):1577-96.

[7] Bruno G, De Micheli A, Frontoni S, Monge L. Società Italiana di Diabetologia-Associazione Medici Diabetologi (SID-AMD) working group on the standards of care for diabetes. Highlights from Italian standards of care for diabetes mellitus. Nutr Metab Cardiovasc Dis 2011;21:302-14.

[8] Kahn SE, Haffner SM, Heise MA, Herman WH, Holman RR, Jones NP, et al. Glycemic durability of rosiglitazone, metformin, or glyburide monotherapy. N Engl J Med 2006;355: 2427-43.

[9] Cleveland Jr JC, Meldrum DR, Cain BS, Banerjee A, Harken AH. Oral sulfonylurea hypoglycemic agents prevent ischemic preconditioning in human myocardium. Two paradoxes revisited. Circulation 1997; 96:29-32.

[10] Klepzig H, Kober G, Matter C, Luus H, Schneider H, Boedeker $\mathrm{KH}$, et al. Sulfonylureas and ischaemic preconditioning; a double-blind, placebo controlled evaluation of glimepiride and glibenclamide. Eur Heart J 1999;20: 439-46.

[11] Horsdal HT, Johnsen SP, Sondergaard F, Jacobsen J, Thomsen RW, Schmitz O, et al. Sulfonylureas and prognosis after myocardial infarction in patients with diabetes: a population-based follow-up study. Diabetes Metab Res Rev 2009; 25:515-22.

[12] Khalangot M, Tronko M, Kravchenko V, Kovtun V. Glibenclamide-related excess in total and cardiovascular mortality risks: data from large Ukrainian observational cohort study. Diabetes Res Clin Pract 2009;86:247-53.

[13] Pantalone KM, Kattan MW, Yu C, Wells BJ, Arrigain S, Jain A, et al. Increase in overall mortality risk in patients with type 2 diabetes receiving glipizide, glyburide, or glimepiride monotherapy vs. metformin: a retrospective analysis. Diabetes Obes Metab 2012;14(9):803-9.

[14] Zeller M, Danchin N, Simon D, Vahanian A, Lorgis L, Cottin Y, et al. Impact of type of preadmission sulfonylureas on mortality and cardiovascular outcomes in diabetic patients with acute myocardial infarction. J Clin Endocrinol Metab 2010;95:4993-5002.
[15] Schramm TK, Gislason GH, Vaag A, Rasmussen JN, Folke F, Hansen ML, et al. Mortality and cardiovascular risk associated with different insulin secretagogues compared with metformin in type 2 diabetes, with or without a previous myocardial infarction: a nationwide study. Eur Heart J 2011; 32:1900-8.

[16] Andersson C, Gislason GH, Jorgensen $C H$, Hansen PR, Vaag A, Sørensen R, et al. Comparable long-term mortality risk associated with individual sulfonylureas in diabetes patients with heart failure. Diabetes Res Clin Pract 2011;94:119-25.

[17] Garratt KN, Brady PA, Hassinger NL, Grill DE, Terzic A, Holmes Jr DR. Sulfonylurea drugs increase early mortality in patients with diabetes mellitus after direct angioplasty for acute myocardial infarction. J Am Coll Cardiol 1999;33: $119-24$.

[18] Zeller M, Danchin N, Simon D, Vahanian A, Lorgis L, Cottin Y, et al. French registry of acute ST-elevation and non-STelevation myocardial infarction investigators. Impact of type of preadmission sulfonylureas on mortality and cardiovascular outcomes in diabetic patients with acute myocardial infarction. J Clin Endocrinol Metab 2010;95(11):4993-5002.

[19] Meinert CL, Knatterud GL, Prout TE, Klimt CR. A study of the effects of hypoglycemic agents on vascular complications in patients with adult-onset diabetes II. Mortality results. Diabetes 1970;19(Suppl.):789-830.

[20] UK Prospective Diabetes Study (UKPDS) Group. Intensive blood-glucose control with sulphonylureas or insulin compared with conventional treatment and risk of complications in patients with type 2 diabetes (UKPDS 33). Lancet 1998;352: 837-53.

[21] The ADVANCE Collaborative Group. Intensive blood glucose control and vascular outcomes in patients with type 2 diabetes. N Engl J Med 2008;358:2560-72.

[22] Yki-Jarvinen H. Thiazolidinediones. N Engl J Med 2004;351: 1106-18.

[23] Dormandy JA, Charbonnel B, Eckland DJA, Erdmann E, MassiBenedetti M, Moules IK, On behalf of the PROactive Investigators, et al. Secondary prevention of macrovascular events in patients with type 2 diabetes in the PROactive Study (PROspective pioglitAzone Clinical Trial In macroVascular Events): a randomized controlled trial. Lancet 2005;366: 1279-89.

[24] Mazzone T, Meyer PM, Feinstein SB, Davidson MH, Kondos GT, D'Agostino RB, et al. Effect of pioglitazone compared with glimepiride on carotid intima-media thickness in type 2 diabetes. a randomized trial. JAMA 2006;296:2572-81.

[25] Nissen SE, Nicholls SJ, Wolski K, Nesto R, Kupfer S, Perez A, et al. For the PERISCOPE Investigators. Comparison of pioglitazone vs. glimepiride on progression of coronary atherosclerosis in patients with type 2 diabetes: the PERISCOPE randomized controlled trial. JAMA 2008;299(13): 1561-73.

[26] Lewis JD, Ferrara A, Peng T, Hedderson M, Bilker WB, Quesenberry Jr CP, et al. Risk of bladder cancer among diabetic patients treated with pioglitazone: interim report of a longitudinal cohort study. Diabetes Care 2011;34(4):916-22.

[27] Li W, Macdonald TM, Mackenzie IS. Pioglitazone and bladder cancer: a propensity score matched cohort study. $\mathrm{Br} \mathrm{J}$ Clin Pharmacol 2012 May 11. http://dx.doi.org/10.1111/j.13652125.2012.04325.x [Epub ahead of print].

[28] Riddle MC, Rosenstok J, Geric JOn behalf of the Insulin Glargine 4002 Study Investigators. The treat-to-target trial. Randomised addition of glargine or human NPH insulin to oral therapy of type 2 diabetic patients. Diabetes Care 2003;26: 3080-6.

[29] Nesto RW, Bell D, Bonow RO, Fonseca V, Grundy SM, Horton ES, et al. Thiazolidinedione use, fluid retention, and 
congestive heart failure: a consensus statement from the American heart association and American diabetes association. Diabetes Care 2004;27:256-63.

[30] Brunn E, Nelson RG, Bennett PH, Imperatore G, Zoppini G, Verlato $G$, et al. Diabetes duration and cause-specific mortality in the Verona diabetes study. Diabetes Care 2000; 23(8):1119-23.

[31] Bo S, Ciccone G, Gancia R, Rosato R, Grassi G, Merletti F, et al. Mortality within the first 10 years of the disease in type
2 diabetic patients. Nutr Metab Cardiovasc Dis 2006;16: 8-12.

[32] McAlpine RR, Morris AD, Emslie-Smith A, James P, Evans MM. The annual incidence of diabetic complications in a population of patients with type 1 and type 2 diabetes. Diabet Med 2005;22:348-52.

[33] The TIDE Trial Investigators. Design, history and results of the tiazolidinedione intervention with vitamin $D$ evaluation (TIDE) randomized controlled trial. Diabetologia 2012;55:36-45. 